\title{
Risk of cancer from occupational exposure to ionising radiation: retrospective cohort study of workers in France, the United Kingdom, and the United States (INWORKS)
}

In this Research paper's (BMJ 2015;351:h5359, doi:10.1136/ bmj.h5359) data supplement, the accompanying legend to figure $\mathrm{S} 1$ has been revised to indicate that the dashed line in the graph represents the fitted model for the excess relative rate (ERR) ๑ BMJ Publishing Group Ltd 2015 per Gy estimated over the entire cumulative dose range. 